\title{
The environmental impact of natural fiber composites through life cycle assessment analysis
}

\begin{abstract}
Growing awareness worldwide toward sustainability has spurred many initiatives to search and apply more environmentally friendly solutions in daily activities. One of the solutions highly investigated currently is for the application of natural fiber composites (NFCs) as a substitution material especially to synthetic composites and other traditional engineering materials. NFC offers many advantages in terms of environmental performance such as renewability, recyclability, and biodegradability, in addition to lower raw material costs and lightweight property. In this chapter, the sustainability performance of natural fiber composites is explained based on the life cycle assessment (LCA) analysis, a methodology formulated to assess the potential environmental impact of products throughout all of their life cycle stages. Among the discussions included are the LCA analysis methodology and collection of research on LCA analysis conducted for natural fiber composites. In addition, a case study on the application of LCA analysis to evaluate the potential environmental impact of automotive products made from natural fiber composites is also included. Eco-indicator 99 method was applied as an impact assessment method in the simplified LCA analysis, based on cradle-to-grave approach. The case study showed that the use of NFCs in hybrid form to produce automotive anti-roll bar components resulted in a lower eco-indicator score, compared to current use of spring steel material. The case study also showcased how the LCA methodology is able to help product designers in performing simplified LCA analysis to assess products' environmental impact holistically throughout their life cycle stages.
\end{abstract}

Keyword: Automotive product; Environmental impact; Life cycle assessment analysis; Natural fiber composites 